\title{
Control de enfermedades fúngicas en la aclimatación de plátano curare enano con el uso Trichoderma harzianum
}

\section{Juan Francisco Cuéllar}

Ingeniero Agrónomo en Desarrollo Rural

Docente-Investigador, Facultad de Ingeniería y Arquitectura

Universidad Católica de El Salvador

Email: jfcuellar@catolica.edu.sv

\section{Colaboradores}

Pedro Vicente Alvergue

Vladimir Alexander Orellana

Estudiantes de Ingeniería Agronómica

Universidad Católica de El Salvador

\section{RESUMEN}

Recepción: 2012-06-13

Aceptacion: 2012-08-15

La investigación consistió en evaluar el uso de Trichoderma en la aclimatación de 1,000 plantas de plátano (curare enano), utilizando 3 gramos de TRICHOZAM ${ }^{\oplus}$, (compuesto por esporas de Trichoderma harzianum $1.25 \times 10^{9}$ UFC/gramo \% p/p 8.3 y materia inerte "Bentonita" \% p/p 91.7) ${ }^{1}$; por cada 200 plantines aclimatados, comparado con un proceso similar, (sin el uso de Trichoderma harzianum), tomando como principal parámetro el porcentaje final de plantas perdidas durante el proceso de aclimatación. La finalidad del estudio fue determinar la eficacia del Trichoderma en el combate contra hongos, en el proceso de aclimatación de plantas de plátano, reproducidas de forma in vitro. Al mismo tiempo se evaluó las plantas infectadas por hongos y el porcentaje de plantas perdidas durante el proceso in vitro, dando como resultado un $10 \%$ de infección por hongos en plantas no tratadas con TRICHOZAM ${ }^{\oplus}$; mientras que en plantas tratadas con TRICHOZAM ${ }^{\circledast}$ la infección fue del $0 \%$. En cuanto a pérdidas finales, en plantas no tratadas el porcentaje fue de $12 \%$, mientras que en las plantas tratadas, el porcentaje fue de $2 \%$. La toma de datos se hizo continuamente desde el día uno de aclimatación, (después de extraída la planta del ambiente controlado), hasta el día cuarenta y seis en el que finaliza este proceso. La muestras se manejaron de igual forma, tomando como única variable el uso de Trichoderma harzianum.

Palabras clave: aclimatación, curare enano, in vitro, Trichoderma harzianum, hongos.

1 Hoja de seguridad de Trichozam. (s.f.). Recuperado de http://cms.fideck.com/userfiles/duwest.com/webmaster/ file/descargas esp/agricola/ Trichozam.pdf $\{$ Consultado 17 de septiembre de 2011\}. 
[ 48 ] Universidad Católica de El Salvador.

\section{ABSTRACT}

This research consists on the evaluation of trichoderm in the acclimatization of 1,000 plantains (curare eneno), using 3 grams of TRICHOZAM ${ }^{\star}$, (composed by spores of Trichoderma har-

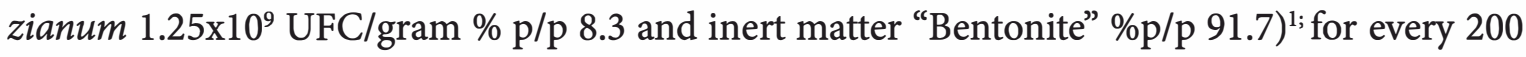
acclimatized seedlings, compared to a similar process (without the use of Trichoderma harzianum), taking as a parameter the percentage of plants that were lost during the acclimatization process. The purpose of the study was to determine the effectiveness of trichoderm fighting the fungi in the acclimatization process of the plantains reproduced in vitro. It was also evaluated the plants infected by fungi and the percentage of plants that were lost during the in vitro process, resulting in a $10 \%$ of fungal infection in plants that were not treated with TRICHOZAM $^{\oplus}$; while the plants that were treated with TRICHOZAM ${ }^{\oplus}$, the level of infection was $0 \%$. Regarding the loss, in the plants that were not treated, the percentage was $12 \%$, whereas in the plants that were treated, the percentage was $2 \%$. The data was gathered continuously since the first day of acclimatization (after the plants were taken from the controlled environment), to the $46^{\text {th }}$ day when the acclimatization process finished. The way the samples were taken was the same; the only variable was the use of Trichoderma harzianum.

Key words : acclimatization, curare enano, in vitro, Trichoderma harzianum, fungi.

\section{INTRODUCCIÓN}

El cultivo in vitro, (término que literalmente significa en vidrio), incluye muchas técnicas destinadas a introducir, multiplicar y regenerar -entre otros recursos- material vegetal o animal en condiciones controladas y asépti$\mathrm{cas}^{2}$. El cultivo de tejidos tiene una metodología muy similar para todas las especies y dentro de las cuales se presentan las siguientes

2 Castillo, A. (2005). Propagación de plantas por cultivo in vitro, una tecnología que nos acompaña hace tiempos, p.1. Recuperado de http:// biociencias.servehttp.com/CENBIO/OPTATIVAS/BIOTECNOLOG\%C $3 \%$. pdf $\{$ Consultado 17 de septiembre de 2011\}. etapas de desarrollo: Establecimiento, elongación, proliferación o micropropagación, enraizamiento, aclimatación o endurecimiento ex vitro ${ }^{3}$.

La importancia de la aclimatación de plátano cultivado in vitro recae en que ésta es una de las partes más importantes en el proceso de cultivo, pues el objetivo de este paso es obtener el mayor porcentaje de sobrevivencia de

3 Valencia, O; Valencia, J; \& Fernández R. (SENA Regional Quíndio). Propagación in-vitro de plátano, banano y frutales de clima frío, p. 1. Recuperado de http://www.agronet.gov.co/www/docs si2/Propagacion\%20 in-vitro\%20de $\% 20$ platano $\% 20$ banano $\% 20 \mathrm{y} \% 20$ frutales $\% 20 \mathrm{de} \% 20$ clima $\% 20$ frio.pdf $\{$ Consultado 30 de agosto de 2011\} 
plantas, aptas para trasplante a suelo $\mathrm{u}$ otro sustrato.

Esta es una fase crítica del proceso a la que se le conoce como endurecimiento, ya que el hombre es quien interviene controlando las condiciones en el invernadero. Dependiendo del manejo que se le dé a la plántula en su adaptación o endurecimiento de cutícula, habrá mayor cantidad de plantas aptas para la siembra de campo. En muchos casos, al no hacer adecuadamente esta etapa el proceso fracasa, ya que acá es donde comúnmente se obtienen las mayores pérdidas ${ }^{4}$. Las plántulas producidas in vitro crecen en un ambiente aséptico y cerrado por lo que son propágulos relativamente delicados.

Para asegurar al máximo el crecimiento de ellas, se suplen sus necesidades con aplicación exógena de nutriente, como aplicar al medio de cultivo la fuente de carbono (azúcar). Éstas son plantas que crecen en condiciones de alta humedad relativa, con baja intensidad de luz y estomas abiertas. Esto hace que al transferirlas a condiciones naturales pierdan agua rápidamente. Además su limitada capacidad fotosintética hace que, en un inicio, la energía demandada sea obtenida por reservas de almidones acumulados durante el cultivo ${ }^{5}$.

4 Ibídem

5 Angarita, A y Perea M. (1986). Micropropagación de plátanos y bananos. Recuperado de http://webapp.ciat. cgiar.org/biotechnology/cultivo tejidos/ capitulo22.pdf. $\{$ Consultado 9 de agosto de 2011\}.
Cabe mencionar que las musáceas no escapan a esta situación. En consecuencia, las hojas no presentan una adecuada capa de cera epicuticular. Además la cutícula es delgada, el mesófilo de empaliada es de escaso desarrollo con grandes espacios intercelulares y baja densidad estomática, lo que las hace susceptibles a la deshidratación. Además, por no realizar fotosíntesis necesitan una baja intensidad lumínica. De ahí que se utilizan diversos medios para la regulación de la luz ${ }^{6}$.

El plátano enano, falso cuerno o curaré enano es una planta que tiene una altura promedio de $2.50 \mathrm{~m}$, lo que la hace más resistente al acame, causado por el viento. Posee un seudo tallo grueso con un diámetro mayor de 25 cm., con abundantes hojas anchas; racimos cortos con un promedio de 40-42 frutos y un promedio de $13.0 \mathrm{~kg}$. de peso. Los frutos poseen buen sabor y son de excelente calidad. El período de floración a cosecha es de 80 - 85 días e inicia entre 10 a 11 meses después de la siembra.

La parte de aclimatación, en el proceso de reproducción in vitro, es la parte más crítica, pues es en donde se tienen mayores pérdidas en cuanto a plantas. De aquí que la idea de la investigación sea evaluar la incidencia del Trichoderma harzianum en la etapa de aclimatación del plátano enano y obtener meno-

6 Zamorano. Evaluación de la producción de plátano de la variedad curaré enano, en función de dos épocas de siembra y tres programas de fertilización. 
res pérdidas de plantas, durante este proceso, por enfermedades fúngicas.

\section{METODOLOGÍA}

Se utilizó un diseño de investigación experimental al azar, en donde se aplicó el tratamiento 1 (aplicación de Trichoderma a 1000 plantas de plátano "curare enano", reproducido de forma in vitro), de las cuales se escogieron plantas al azar. Este tratamiento se comparó con el tratamiento 2 "testigo" (aclimatación de 1000 plantas de plátano "curare enano", reproducido de forma in vitro sin el uso de Trichoderma), en el cual también se utilizaron plantas elegidas al azar, dando como resultado 200 plantines de muestra final. El estudio se llevó a cabo en el centro de aclimatación para plantas reproducidas de forma in vitro de la Universidad Católica de El Salvador (UNICAES), ubicada en Bypass carretera a Metapán y carretera antigua a San Salvador, Santa Ana, El Salvador, C.A. Los datos fueron tomados a partir de una observación continua desde el primer día de aclimatación, (después de extraída la planta del ambiente controlado), hasta el día 46 en el que finalizó el proceso.

\subsection{Materiales utilizados}

1. Trichoderma (15 gramos)

2. Sustrato $(3.25 \mathrm{k})$
3. Bandeja ( 5 bandejas de 200 orificios)

4. Otros recipientes ( 2 beaker, 2 bandejas de secado, bomba de pulverización)

5. Agua (3.25 litros, sin contar agua utilizada en lavados y riegos)

6. Hipoclorito de sodio $(250 \mathrm{ml})$

\subsection{Procedimiento seguido para la aclimatación de plátano enano (curare enano), con el uso de Tri- choderma}

Las cantidades de materiales utilizados fueron diseñados para la aclimatación de 200 plantines. Es decir, una bandeja plástica completa.

\section{a. Selección de plantas a aclimatar}

Las plántulas de plátano (curare enano) fueron extraídas del tubo del medio de cultivo en que se encontraban. Para este paso se utilizaron las muestras del laboratorio de la Universidad Católica de El Salvador.

\section{b. Desinfección del material vegetativo}

Con el uso de lejía, (hipoclorito de sodio al $5 \%$ ), se desinfectó a todos los recipientes utilizados en el proceso de aclimatación. Lo mismo se hizo con la bandeja y demás materiales 
que se utilizaron durante el proceso. Para mejorar el proceso se utilizó agua tibia junto con lejía para una mejor desinfección.

\section{c. Pesaje del sustrato}

El sustrato utilizado en el proceso de aclimatación de plátano enano (curare enano) fue el SUSTRATO CANADIENSE BERGER BM-2. Para la aclimatación de los 200 plantines se utilizó 650 gramos de sustrato, los cuales se depositaron en una bandeja plástica de 200 orificios.

\section{d. Pesaje del Trichoderma}

Para la aclimatación de doscientos plantines (total de un bandeja plástica de 200 orificios) de plátano enano (curare enano), se utilizaron 3 gramos de TRICHOZAM ${ }^{\circledR}$.

\section{e. Dilución de Trichoderma solido en agua}

En el caso de la aclimatación de una bandeja plástica de 200 orificios, (c/u de $15 \mathrm{ml}$.), fueron necesarios 650 gramos de sustrato. Para humectar el sustrato hasta obtener la forma deseada se usaron $650 \mathrm{ml}$. de agua. Por lo tanto se diluyeron 3 gramos de Trichoderma en $650 \mathrm{ml}$. de agua. f. Humectación del sustrato (650 gramos) con la mezcla de agua y Trichoderma (ver paso anterior (650 $\mathrm{ml}$.)

Se vertió la mezcla (Trichoderma y agua) sobre el sustrato a utilizar en el proceso de aclimatación y se amasó para que se humectara de forma equitativa.

\section{g. Efectos del Trichoderma en las raíces de los plantines de plátano (curare enano)}

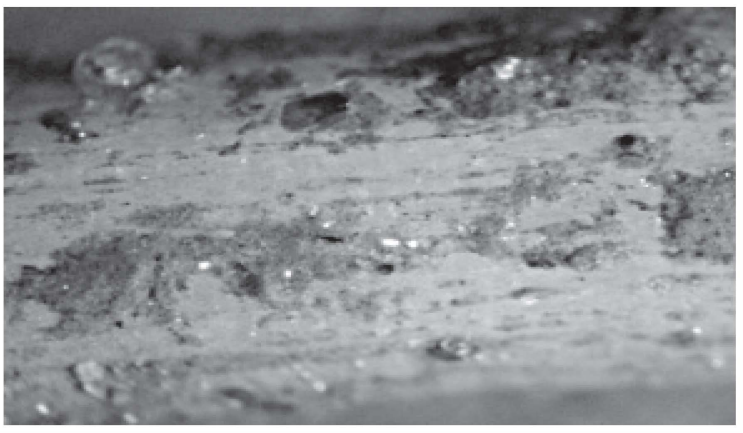

Imagen 7: Foto de raíz de plátano enano (curare enano). Plantas tratadas con fungicida químico.

Fuente: foto tomada el 06/20/11.

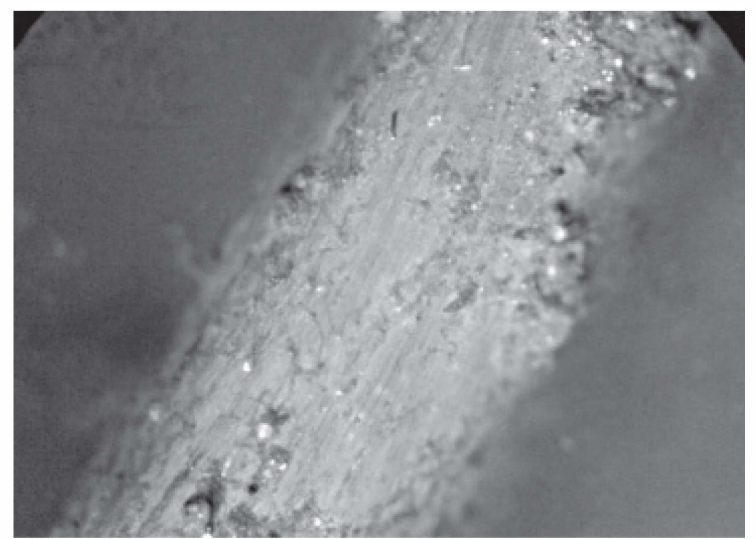

Imagen 8: Foto de raíz de plátano enano (curare enano) cubierta por el hongo Trichoderma harzianum. Vista por medio del microscopio, utilizando el colorante de violeta de Genciana.

Fuente: foto tomada el 06/20/11. 
h. Llenado de bandeja con el sustrato preparado

Se llenó hasta el límite a todas las celdas de la bandeja. En el caso de la bandeja de 200 orificios, (c/u de $15 \mathrm{ml}$.), se debió compactar el sustrato sacudiendo la bandeja, de tal forma que toda la mezcla preparada fuera utilizada.

i. Retiro cuidadoso de la plántula del tubo de ensayo

En este paso se procuró causar el mínimo daño posible a la plántula. Para esto se utilizaron pinzas que permitieron extraer la planta de forma segura.

\section{j. Lavado de raíces con agua tibia} $\left(35^{\circ} \mathrm{C}\right)$ hasta eliminar por completo los residuos de Agar

Las muestras de plátano enano extraídas de su medio de cultivo y en proceso se lavaron para eliminar cualquier residuo de agar en sus raíces.

\section{k. Limpieza de la plántula de materia seca}

Se limpió a las plántulas de cualquier parte del follaje que se observara muerto, lo que facilitó la regeneración de la planta y evitó el uso innecesario de nutrientes en partes muertas de la plántula.

\section{l. Siembra de plántula de plátano ena- no (curare enano) en los orificios de la bandeja con sustrato}

En esta parte se trasladó la plántula a los orificios de la bandeja que contenían sustrato. La plántula permaneció en esas condiciones durante las dos primeras semanas (15 días), del proceso de aclimatación. En esta parte se utilizó un punzón, que ayudó a abrir en el sustrato un orificio donde se sembró la plántula. Luego se juntó nuevamente el sustrato alrededor de la planta, para darle la firmeza necesaria para su buen crecimiento.

Nota: En esta etapa es importante evitar la deshidratación de la plántula, por lo que debe existir un riego continuo de ellas. Las plántulas se regaron, al menos, cada cinco minutos.

\section{m. Mantenimiento de las plántulas sem- bradas en las bandejas de aclimata- ción}

Durante dos semanas, las plántulas se mantuvieron bajo las siguientes condiciones:

$\checkmark \checkmark$ En un estado de sombra y aséptico.

$\checkmark \checkmark$ En condiciones de alta humedad relativa (superiores al 90\%).

Volumen No. 1 Control de enfermedades fúngicas en la aclimatación de plátano enano con el uso Trichoderma harzianum 
$\checkmark \quad$ A una temperatura controlada de $28^{\circ} \mathrm{C}$.

$\checkmark \quad$ Bajo la sombra, con niveles máximos de luz de 33 candelas.

$\checkmark$ Observaciones continúas para verificar el estado de la planta y hacer los arreglos pertinentes.

$\checkmark \quad$ A partir del día ocho se empezó a reducir la humedad relativa de las plántulas, hasta llegar a reducirlo en un $80 \%$ en el día quince.

\section{n. Trasplante de las plántulas de pláta- no del sustrato de aclimatación a bol- sas de vivero}

En esta etapa, en el día dieciséis del proceso de aclimatación, las plantas se sacaron de los orificios de la bandeja y se sembraron de forma individual en bolsas de vivero de $20 \mathrm{x}$ $35 \mathrm{~cm}$. llenas de tierra. Para esto se abrió un orificio en la tierra que contiene la bolsa de vivero, luego se introdujo la planta y se cubrió, nuevamente, con tierra para asegurar su adaptación al nuevo medio.

\section{o. Primera fertilización}

Al momento de trasplantar la planta a la bolsa de vivero, se realizó la fertilización. Se incorporó 3 gramos de fórmula 18-46-0 alrededor de la planta en proceso de aclimatación.

\section{p. Mantenimiento de las plantas sem- bradas en bolsas de vivero}

Durante los siguientes treinta días del proceso, las plantas en aclimatación se mantuvieron bajo las siguientes condiciones:

$\checkmark \quad$ Bajo un sombreador.

$\checkmark \quad$ Riegos diarios.

$\checkmark \quad$ Observaciones para controles de plagas.

\section{q. Finalización del proceso}

Treinta días después de ser trasplantadas a las bolsas de vivero, las plantas estuvieron listas para un nuevo trasplante y manejo en campo.

Nota: En el tratamiento 2 “testigo" se realizó un procedimiento similar, con la única variante de la incorporación de fungicida químico.

El análisis estadístico se realizó mediante una representación de frecuencias estadísticas, a través del programa Microsoft Excel, versión 2010. 


\section{RESULTADOS}

Los resultados obtenidos provienen de 2000 plantas de plátano enano, aclimatadas en el laboratorio de la Universidad Católica de El Salvador, bajo dos tipos de tratamiento diferente: Tratamiento 1: 1000 plantas con el uso de Trichoderma y tratamiento 2: 1000 plantas con uso de fungicida químico en el proceso de aclimatación. Los datos fueron tomados desde el día uno, en la etapa de extracción de la planta del frasco de vidrio de donde fue reproducida, (inicio del proceso de aclimatación), hasta el día cuarenta y seis, etapa en que las plantas debían de ser pasadas a campo, (finalización del proceso de aclimatación).

\subsection{Plantas perdidas por enfermeda- des fúngicas}

Con esta variable se determinó la frecuencia de plantas perdidas en el proceso de aclimatación por enfermedades fúngicas. Se tomó en consideración las plantas muertas por enfermedades fúngicas, indiferencialmente al estado fisiológico de su infección durante el proceso, (del día 1 al día 46 de aclimatación).

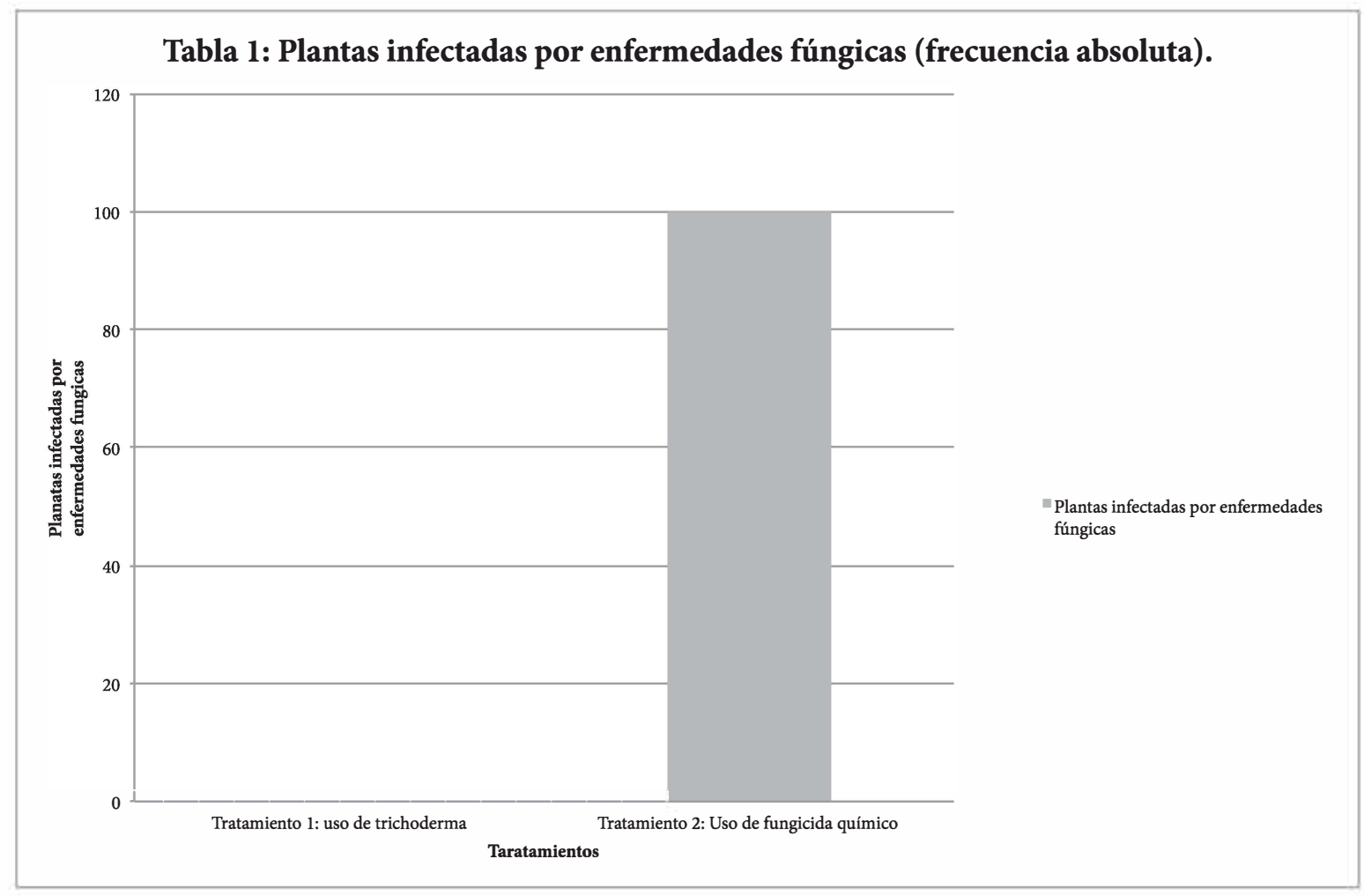

Fuente: Gráfica de elaboración propia a partir de los datos obtenidos. 
3.2. Total de plantas perdidas durante el proceso de aclimatación

Con esta variable se determinó el total de plantas perdidas en el proceso de aclimata- ción. Se tomaron en consideración las plantas muertas, indiferencialmente al estado fisiológico de su infección durante el proceso, (del día 1 al 46 de aclimatación).

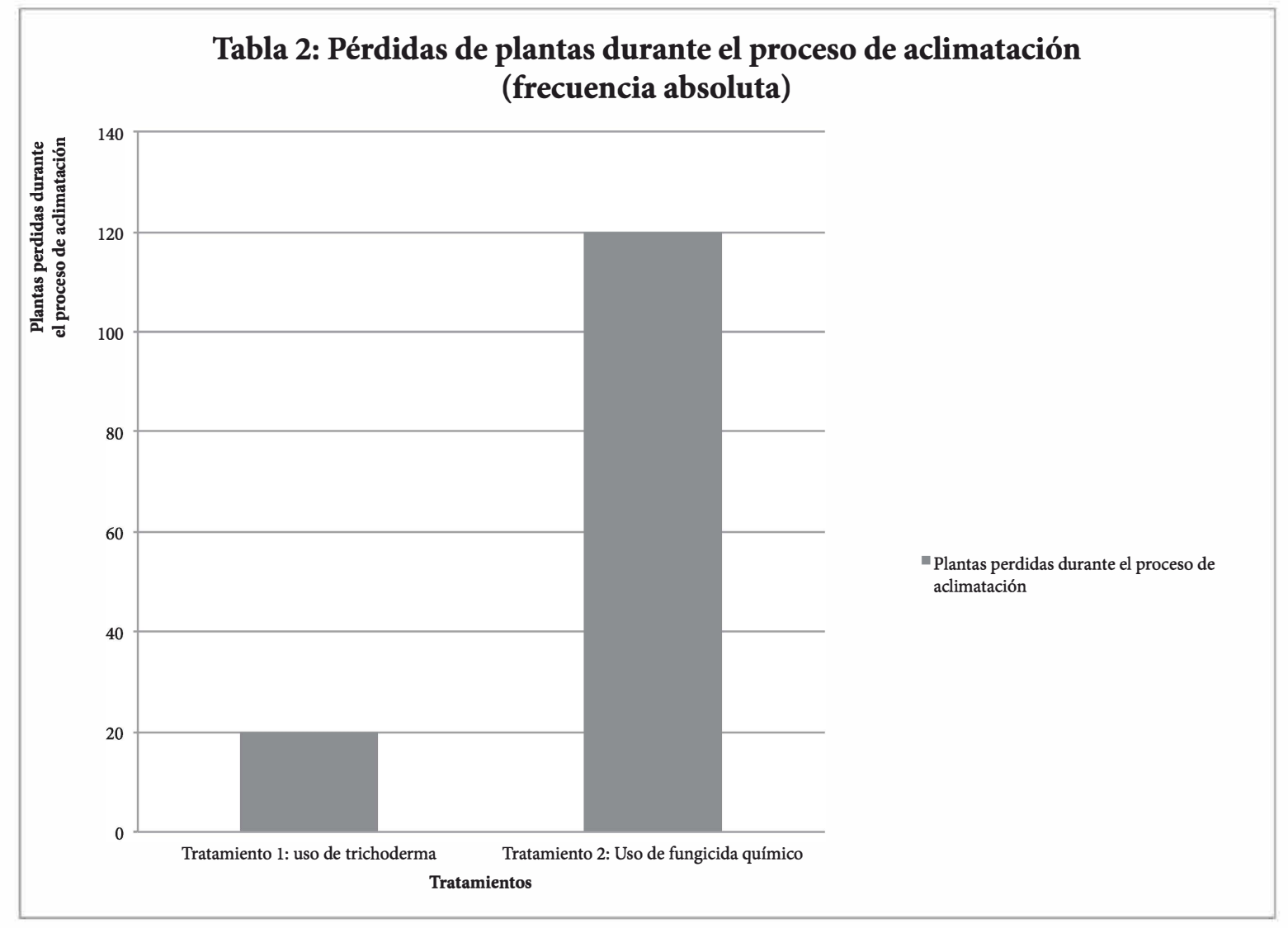

Fuente: Gráfica de elaboración propia a partir de los datos obtenidos.

\section{DISCUSIÓN}

El análisis estadístico de frecuencia absoluta de los datos presentados, anteriormente, demuestra una influencia positiva del uso de Trichoderma harzianum, en la etapa de acli- matación del plátano enano. Los resultados muestran que se obtuvo un $0 \%$ de enfermedades fúngicas en la aclimatación de 1000 plantas de plátano enano tratadas de esta forma. En caso contrario del tratamiento con uso de fungicidas químicos, la infección por hongos fue de $10 \%$ de las plantas aclimatadas. (Hay 
que aclarar que mil plantas fueron aclimatadas bajo el tratamiento 2 "testigo"). El principal hongo que afectó a las plantas sometidas a este tratamiento fue el "Fusarium spp.", principal causante del mal del talluelo.

Además, en la segunda variable analizada, (total de plantas perdidas durante el proceso de aclimatación), se demostró un mayor porcentaje de plantas aclimatadas con éxito, producto del uso de Trichoderma harzianum, con un total de $2 \%$ de plantas perdidas, equivalente a 20 plantas de las 1000 plantas aclimatadas durante la investigación. Mientras que en el tratamiento 2 "sin el uso de Trichoderma harzianum" se obtuvo un total del $12 \%$ de plantas perdidas, lo que equivale a 120 plantas de las 1000 aclimatadas durante la investigación.

Cabe mencionar que las principales causas en la pérdida de las 20 plantas aclimatadas con el uso de Trichoderma recaen, principalmente, en los siguientes puntos:

Manejo inadecuado: Daño mecánico ocasionado a la planta durante el proceso de trasplante.

$\checkmark$ Muerte causada por plagas.

Deshidratación de las plantas.

Se recomienda tener una buena asepsia durante el proceso de aclimatación del plátano, reproducido de forma in vitro, ya que ésta fue una de las principales causas de perdida de plantas durante la evaluación. También se debe tener especial cuidado al momento del trasplante de las plantas, ya que en este paso es en donde más daños mecánicos sufren las plantas, siendo éste procedimiento su principal causa de muerte. Esto sin incluir las enfermedades fúngicas que se eliminaron con el uso de Trichoderma harzianum.

\section{REFERENCIAS BIBLIOGRÁFICAS}

Angarita, A y Perea M. (1986). Micropropagación de plátanos y bananos. Recuperado de http:// webapp.ciat.cgiar.org/biotechnology/cultivo tejidos/ca pitulo22.pdf. \{Consultado 9 de agosto de 2011\}.

Arbelaez A., A; Rodríguez, S.; y Hurtado, A. (2001). Investigaciones sobre Guadua angustifolia realizadas en Colombia (1950-2000). Medellín, Universidad Nacionad de Colombia, sede Medel-

Volumen No. 1 Control de enfermedades fúngicas en la aclimatación de plátano enano con el uso Trichoderma harzianum 
lin. Recuperado de http://www.puj.edu. co/consultoria/archivos/Investigaciones 19502000. pdf $\{$ Consultado 17 de septiembre de 2011\}

Canchignia F., L. R. (2000). Micropropagación de plátano variedad Barragante. Ecuador: Universidad Técnica Estatal de Quevedo, Laboratorio de Biotecnología Vegetal. Recuperado de http://www.uteq.edu.ec/u investi gacion/biotecnologia/3.pdf $\{$ Consultado 25 de septiembre de $2011\}$

Castillo, A. (2005). Propagación de plantas por cultivo in vitro, una tecnología que nos acompaña hace tiempos, p.1. Recuperado de http://biociencias.serve http.com/CENBIO/OPTATIVAS/BIOTECNOLOG\%C3\%.pdf \{Consultado 17 de septiembre de 2011\}.

Hoja de seguridad de Trichozam. (s.f.). Recuperado de http://cms.fideck.com/userfi les/duwest. com/webmaster/file/descargas esp/agricola/Trichozam.pdf \{Consultado 17 de septiembre de $2011\}$

Lardizabal, R. (2007). Manual de produccion de platano de alta intensidad. Cortes, Honduras: FHIA.

McKendrick, D. S. Buenas tareas. Obtenido de Buenas tareas: http://www.buenas tareas.com/ ensayos/Manual-Para-La-Germinacion-In-Vitro/1142397.html \{Consultado el 30 de agosto de $2011\}$

Oliveira, V. (2000). Cultivo in vitro de Gerbera. México D. F.: Grupo Editorial Iberoamérica S.A.

Perea, M. A. (1986). Estrategias para el mejoramiento de bananos y plátanos. Colombia. Revista Augura, ISSN: 0120-4947.

Porquebiotecnologia. (s.f.). Obtenido de porquebiotecnologia: http://www.porque biotecnologia.com.ar/elcuaderno/ecpqb/guia contenidos cuadernos.pdf \{Consultado el 30 de agosto de $2011\}$ 
Ramos, D. A. (2010). Evaluación de cinco sustratos combinados con tres concentraciones de ceniza volcánica en dos tipos de bandeja para la obtención de plántulas de uvilla (Physalis peruvianna L) bajo invernadero. Ecuador: (Tesis) Escuela Superior Politecnica de Chimborazo.

Valencia, O; Valencia, J; y Fernández R. (SENA Regional Quíndio). Propagación in-vitro de plátano, banano y frutales de clima frío, p. 1. Recuperado de http://www.agronet.gov.co/www/ docs si2/Propagacion\%20in-vitro\%20de\%2 0platano, $\% 20$ banano\%20y\%20frutales $\% 20 \mathrm{de} \% 20$ clima\%20frio.pdf $\{$ Consultado 30 de agosto de 2011\}

Ventura, M. Y. (2007). Laboratorio de cultivo in vitro de células. San Salvador, El Salvador.

Zamorano. (2004). Evaluacion de sensibilidad in vitro de Trichozam (trichoderma harzianum) a nueve fungicidas. Honduras: Zamorano.

Zamorano. (2010). Evaluación de la producción de plátano de la variedad curaré enano, en función de dos épocas de siembra y tres programas de fertilización. Honduras: Zamorano. 